\title{
Interplay between Alfvén and magnetosonic waves in compressible magnetohydrodynamics turbulence
}

\author{
N. Andrés ${ }^{1}$, P. Clark di Leoni ${ }^{2,3}$, P. D. Mininni ${ }^{2,3}$, P. Dmitruk ${ }^{2,3}$, F. Sahraoui ${ }^{1}$, and W. H. Matthaeus ${ }^{4}$ \\ ${ }^{1}$ LPP, CNRS, Ecole Polytechnique, UPMC Univ. Paris 06, \\ Univ. Paris-Sud, Observatoire de Paris, Université Paris-Saclay, \\ Sorbonne Universités, PSL Research University, F-91128 Palaiseau, France \\ ${ }^{2}$ Departamento de Física, Facultad de Ciencias Exactas y Naturales, \\ Universidad de Buenos Aires, Ciudad Universitaria, 1428, Buenos Aires, Argentina. \\ 3 Instituto de Física de Buenos Aires, CONICET-UBA, \\ Ciudad Universitaria, 1428, Buenos Aires, Argentina. \\ ${ }^{4}$ Bartol Research Institute and Department of Physics and Astronomy, \\ University of Delaware, Newark, Delaware, USA.
}

(Dated: July 31, 2017)

\begin{abstract}
Using spatio-temporal spectra we show direct evidence of excitation of magnetosonic and Alfvén waves in three-dimensional compressible magnetohydrodynamic turbulence at small Mach numbers. For the plasma pressure dominated regime, or high $\beta$ regime (with $\beta$ the ratio between fluid and magnetic pressure), and for the magnetic pressure dominated regime, or low $\beta$ regime, we study magnetic field fluctuations parallel and perpendicular to a guide magnetic field $\mathbf{B}_{0}$. In the low $\beta$ case we find excitation of compressible and incompressible fluctuations, with a transfer of energy towards Alfvénic modes and to a lesser extent towards magnetosonic modes. In particular, we find signatures of the presence of fast magnetosonic waves in a scenario compatible with that of weak turbulence. In the high $\beta$ case, fast and slow magnetosonic waves are present, with no clear trace of Alfvén waves, and a significant part of the energy is carried by two-dimensional turbulent eddies.
\end{abstract}

\section{INTRODUCTION}

Incompressible magnetohydrodynamics (IMHD) has a wide range of applications as a way to describe the largescale behavior of different types of plasmas, including those of relevance for planetary science, astrophysics, and nuclear fusion science [1] 3 . However, this model is inadequate in those media where density fluctuations cannot be neglected. Examples of these environments are the ionized interstellar medium, some regions of the incoming solar wind, and planetary magnetosheaths [4 6 ] For instance, recent in situ observations have shown that compressibility plays a significant role in the turbulent dynamics of the fast and slow solar wind, in particular by supplying the energy dissipation needed to account for the local heating and particle acceleration of the solar wind [7 11]. Thus, a study of compressible MHD (CMHD) turbulence is essential for a deep understanding of the turbulent dynamics of the solar wind at scales larger than the ion inertial length.

In presence of a uniform magnetic guide field $\mathbf{B}_{0}$, the IMHD model has Alfvén waves as exact non-linear solutions. These transverse and incompressible waves propa- gate along the $\mathbf{B}_{0}$ direction. When a turbulent regime develops in the presence of waves and eddies, two different regimes can be identified depending on the strength of the non-linear coupling, the so-called weak and strong turbulent regimes. In IMHD, the strength of the nonlinear effects is related to the parameter $\chi=\left(k_{\perp} \delta B\right) /\left(k_{\|} B_{0}\right)$, i.e. the ratio between the nonlinear eddy turnover time $\tau_{n l}=k_{\perp} \delta v_{\perp}$ and the linear Alfvén time $\tau_{A}=k_{\|} u_{A}$. In the limit $\chi<<1$, the dynamics is controlled by weakly coupled waves, and perturbation theory can be used to obtain a prediction for the scaling of the energy spectrum 12 15]. When $\chi \gtrsim 1$, waves and eddies coexist with strong coupling, and phenomenological models are often used to study the nonlinear dynamics of turbulent plasmas [16 19]. Note however that even in this case, some exact laws, e.g. the so-called 4/5 law of homogeneous turbulence, can be derived for different fluid approximations of magnetized plasmas [20 28]. It is important to recognize that, in general, the nonlinearity parameter $\chi$ may take on greatly differing values in different regions of $\mathbf{k}$-space.

The existence in IMHD of multiple time scales (the eddy turnover time, the Alfvén time, and the Alfvénic 
crossover time) gives rise to multiple phenomenological models of IMHD turbulence. In the so-called IroshnikovKraichnan (IK) phenomenology [16, 17], the interaction between waves and eddies results in a quenching of the energy transfer towards small scales, which are assumed to be isotropic. This results in a modification of the Kolmogorov energy spectrum from $E(k) \sim k^{-5 / 3}$ 26, 27, 29 31] to $E(k) \sim k^{-3 / 2}$ 32 36]. The anisotropy of IMHD turbulence has been extensively studied in the literature [37 46]. This has resulted in several phenomenological theories that drop the assumption of isotropy but in which the interactions between waves, and of waves with eddies, still play a central role [see, e.g., 18, 19].

Recently, the deep relation between waves and turbulence has been the subject of intensive research [452]. To identify the nature of waves in numerical simulations or experiments the spatio-temporal spectra have been widely used 51 54]. Using direct numerical simulations of the IMHD equations with a uniform magnetic field, Dmitruk and Matthaeus [48] focused on the properties of fluctuations in the frequency domain. The authors found the presence of peaks at the corresponding Alfvén wave frequencies in fully developed turbulent regimes, and nonlinear transfer of energy at wave numbers perpendicular to the mean magnetic field. Meyrand et al. 53 performed three dimensional (3D) numerical simulations of incompressible weak MHD turbulence and found evidence of accumulation of energy in Alfvén waves and in intermittent structures, while Meyrand et al. 51. investigated the transition of turbulence from weak to strong regime. Lugones et al. 52] considered relatively small, medium, and large values of the guide field $\mathbf{B}_{0}$ in IMHD simulations, and found that time decorrelation of Fourier modes is dominated by sweeping effects, and only at large values of $\mathbf{B}_{0}$ and for wave vectors mainly aligned with this field time decorrelations are controlled by the Alfvénic time.

In comparison to IMHD turbulence, CMHD is more intricate due to nonlinear coupling of the velocity, magnetic field, density and pressure fluctuations [see, e.g., 55 57. In the CMHD approximation this emerges as the presence of two additional propagating wave modes that are not present in the IMHD model, namely fast and slow magnetosonic modes. These compressible modes can deeply affect the nonlinear dynamics of turbulent plasmas. Moreover, these modes or their counterparts in kinetic theory were reported using in situ spacecraft mea- surements in the solar wind [see, e.g. 58 60], planetary magnetosheath [61 66] and foreshock regions 67,69$]$.

Different theoretical and numerical efforts have been done to understand the dynamics of compressible flows 24, 25, 57, 70, 71]. Nearly incompressible (NI) MHD theory is an intermediate model between compressible and incompressible descriptions. Using a particular expansion technique, Zank and Matthaeus [57] have derived different NI MHD equations depending on the $\beta$ plasma parameter (ratio between fluid and magnetic pressure). From this NI perspective, one would expect that at high $\beta$ and low Mach number the leading order description would be IMHD 72], with isotropic variances and anisotropic spectra. However, this theoretical predictions are subjected to initial conditions and forcing expressions. In contrast, the low $\beta$ NI MHD theory predicts an anisotropy in both the variances in both the variances and the spatial spectra, which has been observed in the solar wind 73] and confirmed in numerous simulations [see, e.g., 74]. Cho and Lazarian [70] presented a theoretical model for CMHD isothermal turbulence in the low $\beta$ regime, and numerically tested it for moderate spatial resolution $\left(256^{3}\right.$ grid points). The authors separated the different fluctuation modes and reported different theoretical scalings for each branch, namely an anisotropic Kolmogorov spectrum for the Alfvén and slow modes $k_{\perp}^{-5 / 3}$ and an isotropic one $k^{-5 / 3}$ for the fast mode. Using weak turbulence theory 75], Chandran [76] also considered the low $\beta$ regime and derived a set of kinetic equations that provide an approximate description of nonlinear processes in collisionless plasmas. Neglecting the slow magnetosonic branch, Chandran [77] used this model to conclude that three-wave interactions transfer energy to high-frequency fast magnetosonic waves and to a lesser extent to high-frequency Alfvén waves. The author also predicted $\mathrm{a} \sim k^{-7 / 2}$ power spectra for the fast magnetosonic branch for low $\beta$ values. Direct evidence from direct numerical simulations of CMHD turbulence of the excitation of these waves is still lacking, and thus which energy transfer mechanism is dominant is unclear.

The main objective of the present paper is to study the interplay between the different wave modes in a CMHD developed turbulent regime using the spatio-temporal spectrum [78]. This technique allows for direct identification of all wave modes in a turbulent system, and precise quantification of the amount of energy in each mode as a function of the wavenumber. We keep in mind 
that in strong turbulence, much of the energy resides in modes that are not linear eigenmodes, but rather might be described as zero frequency turbulence. Both low and high $\beta$ regimes and small Mach numbers are considered, situations that are relevant for the solar wind and planetary magnetosheaths. The paper is organized as follows: in Section I we present the CMHD model, where in sub-section $\llbracket \mathrm{A}$ we show the set of equations and the normal modes of the CMHD model, in sub-section II we describe the numerical setup used for the study and in sub-section $\llbracket \mathrm{C}$ we briefly explain the spatio-temporal spectrum technique. In Section [II we present our results for both low and high $\beta$. Finally, in Section IV we summarize our main findings.

\section{EQUATIONS, NUMERICAL SIMULATIONS, AND ANALYSIS}

\section{A. Compressible MHD equations}

The 3D CMHD model is given by the mass continuity equation, the momentum equation, the induction equation for the magnetic field, and an equation of state for the plasma, which is assumed here to be polytropic,

$\frac{\partial \rho}{\partial t}+\nabla \cdot(\mathbf{u} \rho)=0$
$\frac{\partial \mathbf{u}}{\partial t}+\mathbf{u} \cdot \nabla \mathbf{u}=-\frac{\boldsymbol{\nabla} p}{\rho}+\frac{\mathbf{J} \times \mathbf{B}}{4 \pi \rho}+\nu^{\prime}\left[\nabla^{2} \mathbf{u}+\frac{1}{3} \boldsymbol{\nabla}(\boldsymbol{\nabla} \cdot \mathbf{u})\right]$

$\frac{\partial \mathbf{B}}{\partial t}=\nabla \times(\mathbf{u} \times \mathbf{B})+\eta^{\prime} \nabla^{2} \mathbf{B}$,

$\frac{p}{\rho^{\gamma}}=$ constant,

where $\mathbf{u}$ is the fluctuating velocity field, $\mathbf{B}=\mathbf{B}_{0}+\mathbf{b}$ is the total magnetic field, and $\rho$ is the density. In addition, $\mathbf{J}=(4 \pi / c) \boldsymbol{\nabla} \times \mathbf{B}$ is the electric current, $p$ the scalar pressure, $\gamma=5 / 3$ the polytropic index, and $\nu^{\prime}$ and $\eta^{\prime}$ are the viscosity and magnetic diffusivity, respectively.

The set of equations (1)-(4) can be written in a dimensionless form in terms of a typical length scale $L_{0}$, a mean scalar density $\rho_{0}$ and pressure $p_{0}$, a typical magnetic field magnitude $b_{r m s}$, and a typical velocity field magnitude $u_{r m s}=b_{r m s} / \sqrt{4 \pi \rho_{0}}$ (i.e., the r.m.s. Alfvén velocity). The resulting dimensionless equations are

$$
\begin{aligned}
& \frac{\partial \rho}{\partial t}+\nabla \cdot(\mathbf{u} \rho)=0, \\
& \frac{\partial \mathbf{u}}{\partial t}+\mathbf{u} \cdot \nabla \mathbf{u}=-\beta \frac{\boldsymbol{\nabla} p}{\rho}+\frac{\mathbf{J} \times \mathbf{B}}{\rho}+\nu\left[\nabla^{2} \mathbf{u}+\frac{1}{3} \boldsymbol{\nabla}(\boldsymbol{\nabla} \cdot \mathbf{u})\right],
\end{aligned}
$$

$\frac{\partial \mathbf{B}}{\partial t}=\nabla \times(\mathbf{u} \times \mathbf{B})+\eta \nabla^{2} \mathbf{B}$,

$\frac{p}{\rho^{\gamma}}=$ constant.

Here, $\nu$ and $\eta$ are the dimensionless viscosity and magnetic diffusivity (i.e., the inverse of Reynolds and magnetic Reynolds numbers) respectively, and $\beta \equiv\left(c_{s} / u_{A}\right)^{2}$ is the plasma beta, i.e., the ratio of plasma pressure to magnetic pressure, with $c_{s}=\sqrt{\gamma p_{0} / \rho_{0}}$ the sound speed and $u_{A}=B_{0} / \sqrt{4 \pi \rho_{0}}$ the Alfvén velocity. The $\beta$ parameter separates two different limiting cases, the magnetic pressure dominated regime $(\beta \ll 1)$ and the plasma pressure dominated regime $(\beta \gg 1)$.

Linearizing equations (5)-(8) around a static equilibrium (i.e., $\mathbf{u}_{0}=0$ ) with a homogeneous magnetic field $\mathbf{B}_{0}=B_{0} \hat{z}$, a constant density $\rho_{0}$, and a constant pressure $p_{0}$, we obtain the dispersion relation $\omega(\mathbf{k})$ of small amplitude waves propagating in the plasma. As usual, the dispersion relation relates the angular frequency $\omega$ of the waves with its wave vector $\mathbf{k}$. It is straightforward [e.g. 79] to show that there are three independent propagating modes (or waves) for a CMHD plasma, which correspond to the so-called Alfvén waves (A), fast (F) and slow (S) magnetosonic waves,

$$
\begin{aligned}
& \omega_{A}^{2}(k)=k_{\|}^{2} u_{A}^{2} \\
& \omega_{F, S}^{2}(k)=k^{2} u_{A}^{2}\left[\frac{(1+\beta)}{2} \pm \sqrt{\frac{(1+\beta)^{2}}{4}-\beta\left(\frac{k_{\|}}{k}\right)^{2}}\right],
\end{aligned}
$$

where $k_{\|}$is the wavenumber component along the external magnetic field, and $k=|\mathbf{k}|=\sqrt{k_{\|}^{2}+k_{\perp}^{2}}$. Alfvén waves are incompressible fluctuations transverse to the magnetic guide field. In the dispersion relation of magnetosonic waves $\omega_{F, S}(k)$, the plus sign on the r.h.s. of the equation (10) corresponds to fast waves, and the minus sign to slow waves. Both fast and slow magnetosonic modes carry density fluctuations, and their magnetic field perturbations have longitudinal and transverse components. Note that for the perpendicular propagation (i.e., $k_{\|}=0$ and $k_{\perp} \neq 0$ ) the Alfvén and slow modes become 
non-propagating modes (i.e., $\omega_{A, S}=0$ ) and are degenerate, but they can be distinguished using their different polarization, since $\delta B_{\|, A}=0$ and $\delta B_{\|, S} \neq 0$ (where $\delta B_{\|}$ is the magnetic fluctuations parallel to the guide field). To these non-propagating solutions one must add the entropy mode $\omega_{E}=0$ characterized by density and entropy fluctuations only. These three non-propagating solutions have their nonlinear counterparts in MHD equilibrium solutions [see, e.g. 80], which are likely to develop in turbulent plasmas. As the main goal of the present paper is to identify the various possible waves and structures in the simulations we adopt the assumption that energy that is concentrated closely to the linear dispersion relation can be explained by linear and weak turbulence theories, while any spread round, or away from, those linear curves is a sign of strong turbulence that requires fully nonlinear theories to be understood.

\section{B. Numerical setup}

The 3D CMHD equations (5)-(8) were numerically solved using the Fourier pseudospectral code GHOST [81, 82 with a new module for compressible flows based on previously developed codes $[83,84$. The scheme used ensures exact energy conservation for the continuous time spatially discrete equation [82] (as well as conservation of all other quadratic invariants in the system). The discrete time integration used is a second-order RungeKutta method. Since computation of the spatio-temporal spectra described below requires a significant amount of data storage, we used moderate linear spatial resolutions $N=512$ in a $3 \mathrm{D}$ periodic box. For simplicity, we used identical dimensionless viscosity and magnetic diffusivity, $\nu=\eta=1 \times 10^{-3}$ (i.e., the magnetic Prandtl number is $P_{m}=1$ ).

The initial state of our simulations corresponds to density, velocity and magnetic fields amplitude fluctuations equal to zero. For all times $t>0$, the velocity field and the magnetic vector potential are forced by a mechanical forcing $\mathbf{F}$ and electromotive forcing $\boldsymbol{\epsilon}$, respectively. The mechanical and electromotive forcings are uncorrelated and they inject neither kinetic nor magnetic helicity. At $t=0$, for each forcing function, a random 3D isotropic field $\mathbf{f}_{\mathbf{k}}$ is generated in Fourier space, by filling the components of all modes in a spherical shell with $1 \leq k \leq 2$ with amplitude $f_{0}$ and a random phase $\phi_{\mathbf{k}}$ for each wave vector $\mathbf{k}$. Here $k=1$ is refers to the longest wavelength in the periodic box. Then, the Fourier coefficients of a forcing with zero divergence are obtained as,

$$
\mathbf{F}_{\mathbf{k}}=\frac{\mathbf{k} \times \mathbf{f}_{\mathbf{k}}}{k}
$$

The same process is repeated to generate $\boldsymbol{\epsilon}_{\mathbf{k}}$ (note that this satisfies the Coulomb gauge used by the code when evolving the vector potential). An amplitude $f_{0}=0.15$ is used for the mechanical and electromotive forcings, and the set of random phases of the two forces are independent. Random phases were also slowly evolving in time, to avoid introducing long-term correlations, but also to prevent introducing very fast time scales. To this end a new set of random phases $\phi_{\mathbf{k}}$ is generated for each forcing function every $1 / 2$ turnover time. Finally, the forcings $\mathbf{F}$ and $\epsilon$ are linearly interpolated from their previous states to the new random states on $1 / 2$ turnover time, and the process is then repeated.

We performed two numerical simulations, both with a weak compressible sonic Mach number $M_{s}=u_{r m s} / c_{s}=$ 0.25 , but with different values of $B_{0}$, and thus different values of $\beta$. In one simulation we used a strong guide magnetic field $B_{0}=8$, which corresponds to $\beta=0.25$. In the other simulation we used a moderated guide field $B_{0}=2$, which corresponds to $\beta=4$. This allowed us to investigate two different regimes, i.e., the magnetic and plasma pressure dominated regimes. Note however that modifying the guide field magnitude results as well in the modification of the nonlinearity parameter $\chi$ (defined above for IMHD turbulence). The simulation with $\beta=4$ corresponds to a nonlinearity parameter (at the driving scale) that is four times higher than the case at $\beta=0.25$. We will return to this point in the discussion Section.

\section{Spatio-temporal spectrum}

The spatio-temporal spectrum allows identification of waves in turbulent flow. The technique consists of calculating the complete spectrum in wavenumber and frequency for all available Fourier modes in a numerical simulation or an experiment [78, 85]. As a result, it can separate between modes that satisfy a given dispersion relation (and are thus associated with waves) from those associated to nonlinear structures or turbulent eddies, and quantify the amount of energy carried by each of them. The method we use does not require the pre-existence 
of wave modes or eddies. Quantifying the relative importance of each of them and understanding the physics that controls it is the main outcome expected from the present analysis. In the following, the spatio-temporal magnetic energy spectral density tensor is defined as

$$
E_{i j}(\mathbf{k}, \omega)=\frac{1}{2} \hat{B}_{i}^{*}(\mathbf{k}, \omega) \hat{B}_{j}(\mathbf{k}, \omega),
$$

where $\hat{B}_{i}(\mathbf{k}, \omega)$ is the Fourier transform in space and time of the $i$-component of the magnetic field $\mathbf{B}(\mathbf{x}, t)$ and where the asterisk implies the complex conjugate. The magnetic energy is associated with the trace of $E_{i j}(\mathbf{k}, \omega)$.

As the external magnetic field $\mathbf{B}_{0}$ in the simulations points in $\hat{z}$, in practice we will consider either $i=j=y$ or $i=j=z$, to identify different waves based on their polarization (either transverse or longitudinal with respect to the guide field). It is worth mentioning that spatio-temporal spectra have been used before in numerical simulations and experiments of rotating turbulence [86], stratified turbulence [87], quantum turbulence [88], and IMHD turbulence simulations [51, 53, 89] and in spacecraft observations [85, 90]. In the present paper we use the technique to investigate the interplay between Alfvén and magnetosonic waves in CMHD turbulence.

In all cases, the temporal extent of the data used to calculate the spatio-temporal spectra was longer than at least one period of the slowest wave in the system, and the temporal data cadence was at least twice as fast as the fastest wave. The emergence of fluctuations occurring on very long time scales, corresponding to $1 / f$ noise in the power frequency spectrum, have been observed in systems such as IMHD with a background magnetic field or in rotating fluid turbulence [64, 91 95]. However, in the present paper we emphasize the wave modes at higher frequencies and not the dominance by $1 / f$ noise at long time scales.

\section{NUMERICAL RESULTS AND DISCUSSION}

\section{A. Low $\beta$ regime}

Reduced spatial spectra are obtained from the general spatio-temporal spectra by integration over all frequencies and over all wave vectors in spherical shells of radius $k$. As an example, for the magnetic energy the spatial

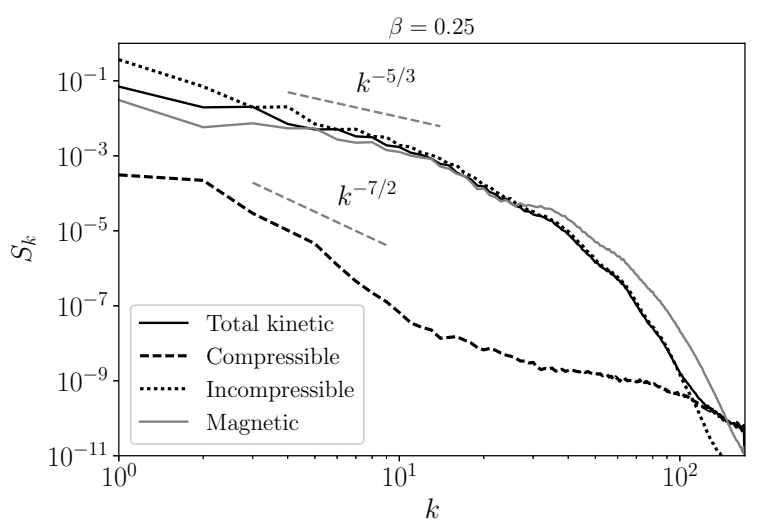

Figure 1. Spatial spectrum $S_{k}$ of the total magnetic and kinetic energy (in solid gray and black lines, respectively). The dotted and dashed lines correspond to the kinetic energy spectra of the incompressible and compressible components of the flow, respectively. Two scaling laws, $\sim k^{-5 / 3}$ and $\sim k^{-7 / 2}$ are shown as references.

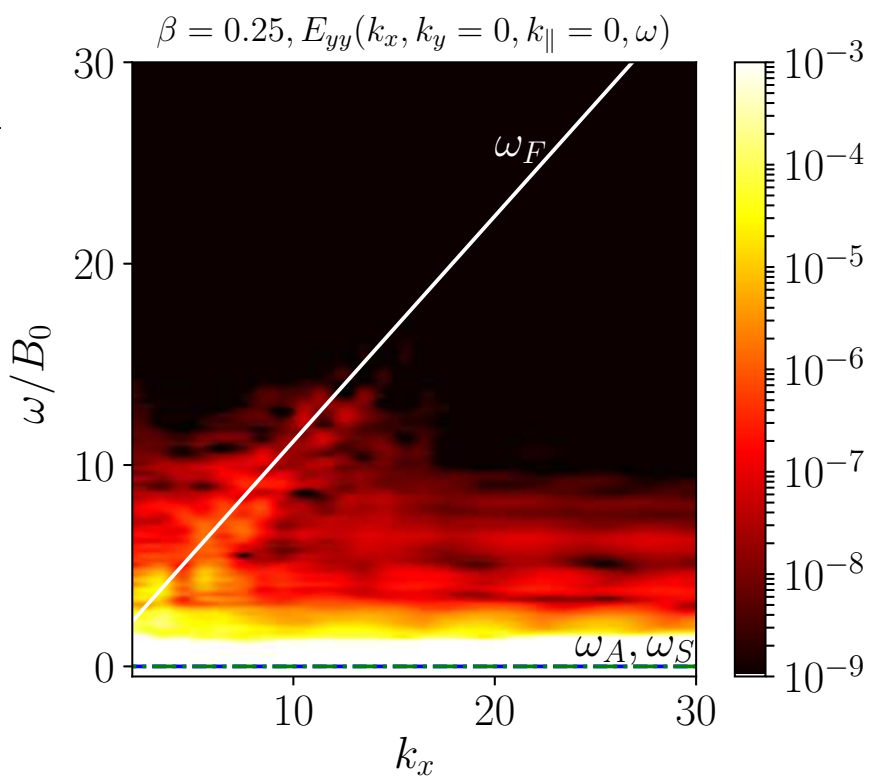

Figure 2. (Color online) Spatio-temporal spectrum $E_{y y}\left(k_{x}, k_{y}=0, k_{\|}=0, \omega\right)$ for the magnetic field fluctuations parallel perpendicular to $\mathbf{B}_{0}$, for $\beta=0.25$. The dashed, solid, and dash-dotted lines correspond to the linear dispersion relationd of Alfvén waves $\omega_{A}$, of fast magnetosonic waves $\omega_{F}$, and of slow magnetosonic waves $\omega_{S}$, respectively.

isotropic (omnidirectional) spectrum satisfies,

$$
S_{k}(k)=\sum_{\omega} \sum_{k \leq|\mathbf{k}|<k+1}\left[E_{x x}(\mathbf{k}, \omega)+E_{y y}(\mathbf{k}, \omega)+E_{z z}(\mathbf{k}, \omega)\right] .
$$



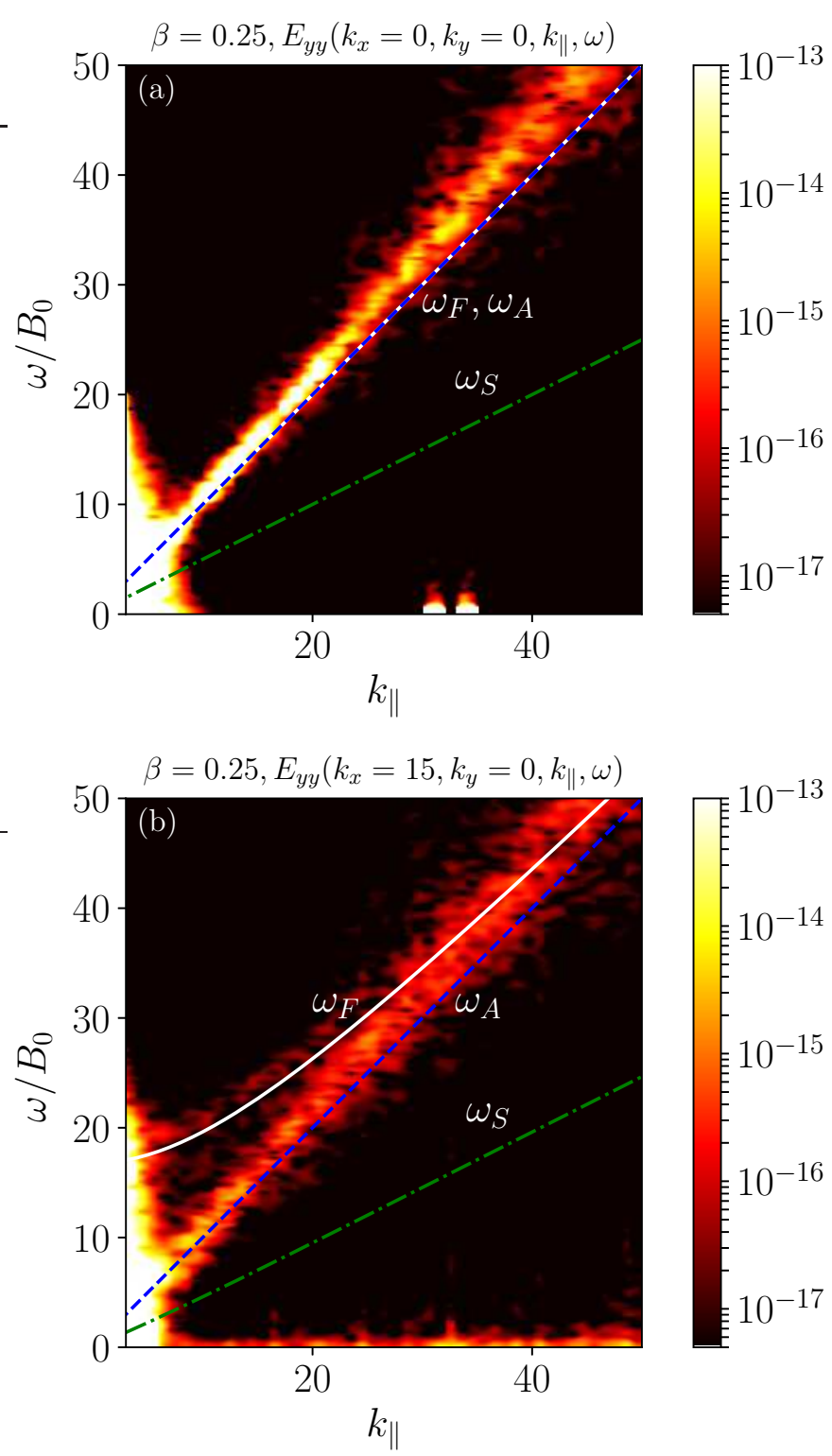

Figure 3. (Color online) Spatio-temporal spectrum $E_{y y}\left(k_{x}=\right.$ $\left.0, k_{y}, k_{\|}, \omega\right)$ for the magnetic field fluctuations perpendicular to $\mathbf{B}_{0}$, for $\beta=0.25$. The spectrum is shown as a function of $\omega$ and $k_{\|}$for fixed $k_{y}=0$ (a) and $k_{y}=15$ (b). The dashed, solid, and dash-dotted lines correspond to the linear dispersion relationd of Alfvén waves $\omega_{A}$, of fast magnetosonic waves $\omega_{F}$, and of slow magnetosonic waves $\omega_{S}$, respectively. For $k_{\perp}=0$ the Aflvén and fast branches coincide.

Similarly, we computed the spatial isotropic spectrum for the kinetic energy. Besides, we computed the compressible and incompressible kinetic spectrum of the flow using the usual Helmholtz decomposition [see, e.g. 96]. In Fig. 1 we show the spatial energy spectra $S_{k}$ of the kinetic and the magnetic energy for the simulation with $\beta=0.25$. We also show the power spectra of the com- pressible and incompressible components of the velocity field.

An inertial range compatible with $\mathrm{a} \sim k^{-5 / 3}$ can be observed in Fig. 1 for the total kinetic energy, the incompressible kinetic energy, and the magnetic energy. The compressible kinetic energy spectrum is weaker and steeper; the $\sim k^{-7 / 2}$ scaling predicted by Chandran 77 is shown for reference. A detailed study of these scaling laws would require larger spatial resolutions, which are outside the scope of this work. Note also that while the vast majority of the kinetic energy is located in its incompressible component, it is known that the small compressible component can still affect the flow dynamics in this regime. For example, direct numerical simulations performed with the same Mach and $\beta$ numbers show that proton acceleration is significantly enhanced when compared to the incompressible case [8].

Spatial analysis alone cannot fully determine the presence of Alfvén or magnetosonic waves, much less determine which (if any) dominates the dynamics; to do this we must turn to spatio-temporal analysis. Fig. 2 shows the spatio-temporal spectrum of the perpendicular magnetic field fluctuations $E_{y y}\left(k_{x}, k_{y}=0, k_{\|}=0, \omega\right)$ for fixed $k_{y}=k_{\|}=0$ for the same simulation as in Fig. 1)(since the spatio-temporal spectrum is four dimensional, we fix two components of $\mathbf{k}$ to plot the remaining component against the frequency). The dispersion relations for Alfvén and magnetosonic waves given by equations (9) and (10) are shown in dashed, dash-dotted, and dotted lines, respectively. The energy accumulates mainly for low $\omega / B_{0}$ $(\lesssim 4)$ and $k_{\|}=0$, i.e., in two-dimensional (2D) modes, which correspond to turbulent eddies and which is to be expected for IMHD turbulence with a guide field. Fig. 3 shows the spatio-temporal spectrum of the perpendicular magnetic field fluctuations $E_{y y}\left(k_{x}=0, k_{y}, k_{\|}, \omega\right)$, for fixed values of $k_{y}=0$ (Fig. 3(a)) and $k_{y}=15$ (Fig. 3(b)). In this case, energy accumulates mostly in modes with low $k_{\|}$and low $\omega / B_{0}$, typically $k_{\|} \lesssim 5$ and $\omega / B_{0} \lesssim 20$. Fig. 2 and Fig. 3 are compatible with the NI MHD theory for low $\beta$, where the leading order description is two-dimensional with compressible corrections. Thus, as in the case of IMHD turbulence [44, 46], the presence of a strong guide field produces strong bidimensional components even in the presence of weak compressibility.

For modes with $k_{\|} \gtrsim 5$, energy in Fig. [3(a) then accumulates around the Alfvén wave branch $\omega_{A}$ (note that for $k_{\perp}=0$, the Alfvén and fast branches overlap), while 


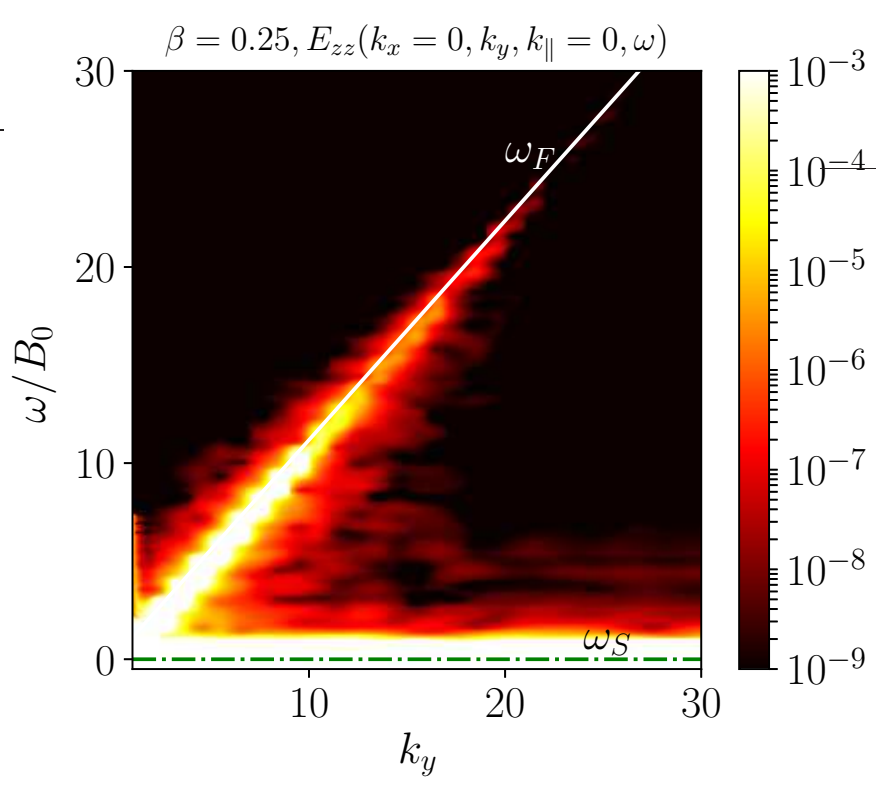

Figure 4. (Color online) Spatio-temporal spectra $E_{z z}\left(k_{x}=\right.$ $\left.0, k_{y}, k_{\|}=0, \omega\right)$ for the magnetic field fluctuations parallel to $\mathbf{B}_{0}$, for $\beta=0.25$. The solid and dash-dotted lines correspond to the linear dispersion relationd of of fast magnetosonic waves $\omega_{F}$, and of slow magnetosonic waves $\omega_{S}$, respectively.

Fig. 3(b) some energy also present in the vicinity of the fast magnetosonic branch $\omega_{F}$ and along $\omega=0$. Both Figures do not show energy spread along the slow magnetosonic branch $\omega_{S}$. In other words, energy in high frequency modes $\left(\omega>0\right.$ and with $\left.k_{\|} \gtrsim 5\right)$ accumulates near the dispersion relation of the fastest waves, in agreement with predictions from weak turbulence [76, 77]. At high parallel wavenumbers energy accumulation deviates slightly from the linear dispersion relations, but is still concentrated around specific modes, indicating possible coupling of fast magnetosonic and Alfvén waves, or nonlinear corrections to the dispersion relations. In contrast with what was previously suggested [70], fast magnetosonic waves are not suppressed by Alfvén waves, but they do not dominate the dynamics either as predicted using weak wave turbulence theory [76, 77].

Fast magnetosonic waves can be separated from the Alfvén waves by looking at the spatio-temporal spectrum of parallel magnetic field fluctuations $E_{z z}\left(k_{x}=0, k_{y}, k_{\|}=0, \omega\right)$, shown in Fig. 4. The Alfvén waves do not contribute to the parallel component of the magnetic field energy since their magnetic perturbations are perpendicular to the guide field. In Fig. 4 energy accumulates in two regions: at high

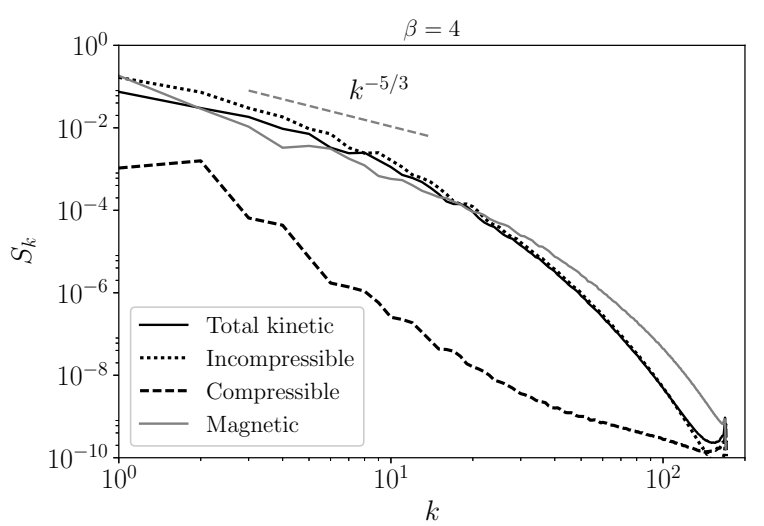

Figure 5. Spatial spectrum $S_{k}$ of the total magnetic and kinetic energy (in solid gray and black lines, respectively). The dotted and dashed lines correspond to the power spectra of incompressible and compressible components of the flow, respectively. $\mathrm{A} \sim k^{-5 / 3}$ scaling is shown as reference.

frequency near the fast magnetosonic branch, and at low frequency near $\omega=0$ modes. Note that the spread around the linear dispersion relations curves is likely to be caused by nonlinear effects. It is worth noticing that, unlike in Fig. 3(a), energy in Fig. [ does not show any shift toward higher frequency than the linear dispersion relation of the fast mode.

\section{B. High $\beta$ regime}

In Fig. [5 we show the spatial energy spectra $S_{k}$ of the kinetic and the magnetic energy for the simulation with $\beta=4$; we also show the energy spectra of the compressible and incompressible components of the velocity field. An inertial range roughly compatible with $\sim k^{-5 / 3}$ is observed for the total kinetic energy, the incompressible kinetic energy, and the magnetic energy. No discernible scaling is present in the compressible kinetic energy spectrum. Once again, the vast majority of the kinetic energy is in the incompressible component of the flow.

To determine the presence of waves in the higher frequency part of the turbulent flow we turn once more to the spatio-temporal spectrum. Fig. [6 and Fig. 7 show the spatio-temporal spectrum of the perpendicular magnetic field fluctuations $E_{y y}\left(k_{x}=0, k_{y}=0, k_{\|}, \omega\right)$, and the spectrum of parallel magnetic field fluctuations $E_{z z}\left(k_{x}=0, k_{y}, k_{\|}=0, \omega\right)$, respectively. The dispersion relations given by equations (9)-(10) are in dashed, dash- 


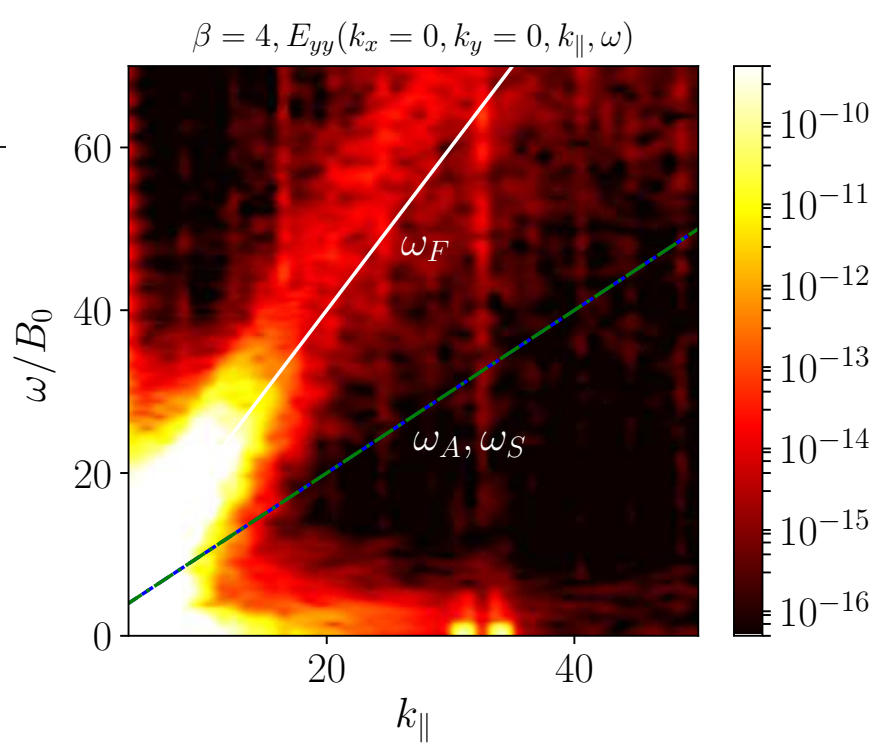

Figure 6. (Color online) Spatio-temporal spectrum $E_{y y}\left(k_{x}=\right.$ $\left.0, k_{y}=0, k_{\|}, \omega\right)$ of the magnetic field fluctuations perpendicular to $\mathbf{B}_{0}$, for the run with $\beta=4$. The dashed, solid, and dash-dotted lines correspond to the linear dispersion relation of Alfvén waves $\omega_{A}$, of fast magnetosonic waves $\omega_{F}$, and of slow magnetsonic waves $\omega_{S}$, respectively (in this case, for $k_{\perp}=0$ the dispersion relations of slow and Alfvén waves coincide).

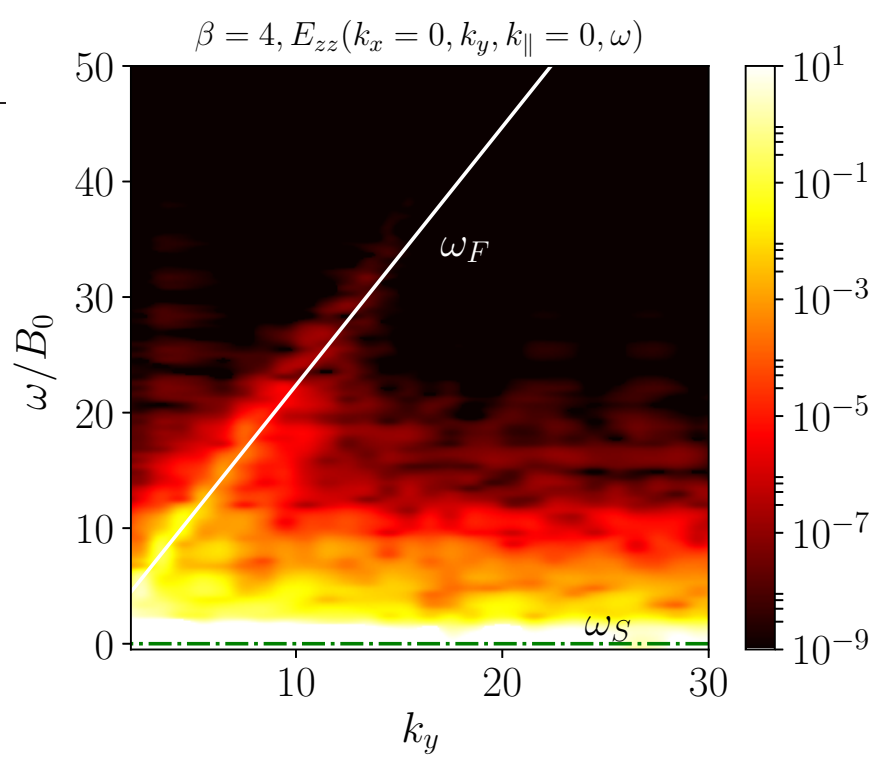

Figure 7. (Color online) Spatio-temporal spectrum $E_{z z}\left(k_{x}=\right.$ $\left.0, k_{y}, k_{\|}=0, \omega\right)$ of the magnetic field fluctuations parallel to $\mathbf{B}_{0}$, for $\beta=4$. The solid and dash-dotted lines correspond to the linear dispersion relation of fast magnetosonic waves $\omega_{F}$, and of slow magnetsonic waves $\omega_{S}$, respectively dotted, and dotted lines. Fig. [6 shows that for frequencies $\omega / B_{0} \gtrsim 10$ and $k_{\|} \gtrsim 5$, the only wave modes that are now excited are the fast magnetosonic ones, and no apparent traces of Alfvén waves, which coincide with the slow mode in this case. Fig. 6 shows also a smaller amount of energy near the $\omega=0$ (and $k_{\|} \neq 0$ ) modes. Fig. 7 shows that most of the energy lies along the slow mode $\omega_{S}$ curve, and a smaller fraction of energy follow the fast mode curve $\omega_{F}$. The spread around those curves is likely to be due to stronger nonlinear interactions that can generate $2 \mathrm{D}$ structures that can coincide with the curve $\omega_{S}$ in Fig. 7 .

It is worth mentioning that, for an IMHD run with a guide field $\mathbf{B}_{0}=2$ (not show here) we obtain a similar result to Fig. 7, without the fast magnetosonic trace. This supports the dominance of the 2D (incompressible) structures in Fig. 7 rather than the non-propagating (compressible) mode $\omega_{S}$. Furthermore, the absence of Alfvén waves might be due to the weak magnetic guide field used $\left(\mathbf{B}_{0}=2\right)$, as already was found in IMHD simulations 52]. Therefore, in the high $\beta$ regime, fast magnetosonic modes dominate the dynamics (over the Alfvén waves) at high frequencies and wavenumbers. However, as we mentioned above, we emphasize that the system in its entirety is dominated mainly by the contributions of $2 \mathrm{D}$ modes related to turbulent eddies and non-propagating slow (or entropy) modes $\left(k_{\|}=0\right.$ and $\omega=0$ ).

Despite the fact that fast magnetosonic waves concentrate most of the energy in the waves at high frequency, their contribution to the total energy in the system is bounded by the small fraction of energy in compressible

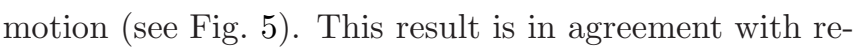
cent 3D Landau-fluid simulations [54]. The reason for observing fast modes (rather than Alfvén modes) in the high plasma $\beta$ regime remains unclear. We speculate that they might have been favored by the isotropic forcing used in our simulations (the fast modes being the only isotropic modes [70]). Future numerical simulations with a different (anisotropic) forcing will be needed be needed to unambiguously answer this question.

Another question is whether the different results obtained in the low and high plasma $\beta$ regimes are actually due to the change in the plasma $\beta$ or to that of the nonlinear parameter $\chi$ discussed above, as demonstrated in recent Landau-fluid simulations [54, 97]. The fact that the simulation in the low $\beta$ case corresponds to a nonlinearity parameter that is four time smaller than that of 
the high $\beta$ case may explain the observations of different branches of the linear modes in the former case. It may also explain the broad accumulation of energy around the fast magnetosonic waves in the case of high $\beta$ (Fig. 6) in comparison to that around Alfvén waves in the low $\beta$ case (Fig. 4). However, more numerical simulations are required to answer this question.

\section{CONCLUSIONS}

We used spatio-temporal spectra of different magnetic field components to study waves in compressible MHD turbulence at low and high $\beta$ regimes. In the magnetic pressure dominated regime, we showed direct evidence of the presence of fast magnetosonic and Alfvén waves. In particular, we found wavenumber scaling for the spatial spectra compatible with theoretical predictions. We also found that the energy transfer is dominated by the Alfvénic or the incompressible fluctuations, and to a lesser extent by fast magnetosonic fluctuations (specially in the perpendicular direction). Although the role of magnetosonic waves is not as important as predicted by some weak wave turbulence theories [76, 77], they are not negligible. Moreover, the results confirm that the fastest waves in the system concentrate a non-negligible fraction of the energy at high frequency (even for moderate values of the sonic Mach number), and can thus have a role in the dynamics, with implications for particle acceleration and other processes in the solar wind.

In the high $\beta$ regime, at high frequency only fast magnetosonic waves were present, with no clear trace of Alfvén waves. At low frequency, 2D turbulent eddies and non-propagating slow (or entropy) modes may co-exist and seem to carry most of the turbulent energy. This regime is thus similar to that of IMHD with a weak magnetic guide field. The questions as to how the dynamics changes when increasing the magnetic guide field and the Mach number, or when fixing the same plasma $\beta$ and modifying the $\chi$ parameter at the driving scale will be addressed in future studies.

\section{ACKNOWLEDGMENTS}

N.A. is supported through an École Polytechnique Postdoctoral Fellowship and by LABEX Plas@Par through a grant managed by the Agence Nationale de la Recherche (ANR), as part of the program "Investissements d'Avenir" under the reference ANR-11IDEX-0004-02. F.S. and N.A. acknowledge financial support from Programme National Soleil-Terre (PNST). P.C., P.D.M. and P.D. acknowledge support from UBACYT Grant No. 20020130100738BA, and PICT Grants Nos. 2011-1529 and 2015-3530. W.H.M. is supported in part by the NASA Heliophysics Grand Challenge (NNX14AI63G), LWS (NNX14AI63G) and Heliophysics GI (NNX17AB79G) programs.
[1] E. Priest, Solar Magnetohydrodynamics (D. Reidel Publishing Company, 1982).

[2] A. Pouquet, Magnetohydrodynamic turbulence, edited by J. P. Zahn and J. Zinn-Justin (Session XLVII, Elseiver, 1993) pp. 139-227.

[3] D. Biskamp, Nonlinear Magnetohydrodynamics (Cambridge Univ. Press, 1997).

[4] J. W. Armstrong, J. M. Cordes, and B. J. Rickett, Nature 291, 561 (1981).

[5] B. Bavassano, M. Dobrowolny, G. Fanfoni, F. Mariani, and N. F. Ness, Solar Physics 78, 373 (1982).

[6] R. Bruno and V. Carbone, Living Reviews in Solar Physics 2, 4 (2005).

[7] V. Carbone, R. Marino, L. Sorriso-Valvo, A. Noullez, and R. Bruno, Phys. Rev. Lett. 103, 061102 (2009).
[8] C. A. González, P. Dmitruk, P. D. Mininni, and W. H. Matthaeus, Physics of Plasmas 23, 082305 (2016).

[9] S. Banerjee, L. Z. Hadid, F. Sahraoui, and S. Galtier, The Astrophysical Journal Letters 829, L27 (2016).

[10] L. Z. Hadid, F. Sahraoui, and S. Galtier, ApJ 9, 838 (2017a).

[11] L. Z. Hadid, F. Sahraoui, and S. Galtier, Manuscript submitted (2017b).

[12] V. E. Zakharov, J. Appl. Mech. Tech. Phys. 6, 22 (1965).

[13] S. Galtier, S. V. Nazarenko, A. C. Newell, and A. Pouquet, J. Plasma Phys. 63, 447 (2000).

[14] S. Galtier, Phys. Rev. E 68, 015301 (20043).

[15] S. Nazarenko, Wave Turbulence (Springer, 2011).

[16] P. S. Iroshnikov, Astronomicheskii Zhurnal 40, 742 (1963).

[17] R. H. Kraichnan, Phys. Fluids 8, 1385 (1965). 
[18] J. C. Higdon, ApJ 285, 109 (1984).

[19] P. Goldreich and S. Sridhar, ApJ 438, 438 (1995).

[20] T. von Kármán and L. Howarth, Proc. R. Soc. London 164, 192 (1938).

[21] S. Chandrasekhar, Proc. R. Soc. London, Ser A 204, 435 (1951).

[22] H. Politano and A. Pouquet, Phys. Rev. E 57, R21 (1998a).

[23] D. O. Gómez, S. M. Mahajan, and P. Dmitruk, Phys. Plasmas 15, 102303 (2008).

[24] S. Galtier and S. Banerjee, Phys. Rev. Lett. 107, 134501 (2011).

[25] B. Banerjee and S. Galtier, Phys. Rev. E 87, 013019 (2013).

[26] N. Andrés, P. Mininni, P. Dmitruk, and D. O. Gómez, Phys. Rev. E 93, 063202 (2016a).

[27] N. Andrés, S. Galtier, and F. Sahraoui, Phys. Rev. E 94, 063206 (2016b).

[28] N. Andrés and F. Sahraoui, Manuscript submitted (2017).

[29] W. H. Matthaeus and M. L. Goldstein, J. Geophys. Res. 87, 6011 (1982).

[30] F. Sahraoui, M. L. Goldstein, P. Robert, and Y. V. Khotyaintsev, Phys. Rev. Lett. 102, 231102 (2009).

[31] N. Andrés, L. N. Martin, P. Dmitruk, and D. O. Gómez, Phys. Plasmas 21, 072904 (2014).

[32] F. L. Scarf, J. H. Wolfe, and R. W. Silva, J. Geophys. Res. 72, 993 (1967).

[33] W. Matthaeus and Y. Zhou, Phys. Fluids B 1, 1929 (1989).

[34] C. S. Ng, A. Bhattacharjee, D. Munsi, P. A. Isenberg, and C. W. Smith, J. Geophys. Res. 115 (2010).

[35] J. J. Podesta, D. A. Roberts, and M. L. Goldstein, Astrophys. J. 664, 543 (2007).

[36] R. Bruno, V. Carbone, B. Bavassano, and L. SorrisoValvo, Adv. Space Res. 35, 939 (2005).

[37] D. Montgomery and L. Turner, The Physics of Fluids 24, 825 (1981).

[38] J. V. Shebalin, W. H. Matthaeus, and D. Montgomery, Journal of Plasma Physics 29, 525 (1983).

[39] S. Oughton, E. R. Priest, and W. H. Matthaeus, Journal of Fluid Mechanics 280, 95 (1994).

[40] D. Montgomery and W. H. Matthaeus, ApJ 447, 706 (1995).

[41] W. H. Matthaeus, S. Ghosh, S. Oughton, and D. A. Roberts, Journal of Geophysical Research: Space Physics 101, 7619 (1996).

[42] M. L. Goldstein and D. A. Roberts, Phys. Plasmas 6, 4154 (1999).

[43] A. Bhattacharjee and C. S. Ng, ApJ 548, 318 (2001).

[44] A. Alexakis, Phys. Rev. E 84, 056330 (2011).
[45] P. Mininni, Ann. Rev. Fluid Mech. 43, 377-397 (2011).

[46] N. E. Sujovolsky and P. D. Mininni, Phys. Rev. Fluids 1, 054407 (2016).

[47] E. Falcon, C. Laroche, and S. Fauve, Phys. Rev. Lett. 98, 094503 (2007).

[48] P. Dmitruk and W. H. Matthaeus, Physics of Plasmas 16, 062304 (2009).

[49] P. D. Mininni, P. Dmitruk, W. H. Matthaeus, and A. Pouquet, Phys. Rev. E 83, 016309 (2011).

[50] R. Meyrand and S. Galtier, Phys. Rev. Lett. 111, 264501 (2013).

[51] R. Meyrand, S. Galtier, and K. H. Kiyani, Phys. Rev. Lett. 116, 105002 (2016).

[52] R. Lugones, P. Dmitruk, P. D. Mininni, M. Wan, and W. H. Matthaeus, Physics of Plasmas 23, 112304 (2016).

[53] R. Meyrand, K. H. Kiyani, and S. Galtier, Journal of Fluid Mechanics 770 (2015).

[54] S. Kobayashi, F. Sahraoui, T. Passot, D. Laveder, P. L. Sulem, S. Y. Huang, P. Henri, and R. Smets, The Astrophysical Journal 839, 122 (2017).

[55] G. P. Zank and W. H. Matthaeus, Physical review letters 64, 1243 (1990).

[56] G. Zank and W. Matthaeus, J. Plasma Phys 48, 85 (1992).

[57] G. P. Zank and W. H. Matthaeus, Physics of Fluids A: Fluid Dynamics 5, 257 (1993).

[58] K. G. Klein, G. G. Howes, J. M. TenBarge, and J. J. Podesta, The Astrophysical Journal 785, 138 (2014).

[59] L. B. Wilson, in Low-Frequency Waves in Space Plasmas (John Wiley and Sons, Inc, 2016) pp. 269-291.

[60] L. Ofman, Low-Frequency Waves in Space Plasmas 216, 243 (2016).

[61] F. Sahraoui, J. L. Pinçon, G. Belmont, L. Rezeau, N. Cornilleau-Wehrlin, P. Robert, L. Mellul, J. M. Bosqued, A. Balogh, P. Canu, and G. Chanteur, Journal of Geophysical Research: Space Physics 108 (2003), 1335.

[62] F. Sahraoui, G. Belmont, L. Rezeau, N. CornilleauWehrlin, J. L. Pinçon, and A. Balogh, Phys. Rev. Lett. 96, 075002 (2006).

[63] N. Romanelli, C. Bertucci, D. Gómez, C. Mazelle, and M. Delva, Planetary and Space Science 76, 1 (2013).

[64] L. Z. Hadid, F. Sahraoui, K. H. Kiyani, A. Retinò, R. Modolo, P. Canu, A. Masters, and M. K. Dougherty, The Astrophysical Journal Letters 813, L29 (2015).

[65] S. Huang, Z. Yuan, B. Ni, M. Zhou, H. Fu, S. Fu, X. Deng, Y. Pang, H. Li, D. Wang, et al., Journal of Atmospheric and Solar-Terrestrial Physics 129, 119 (2015).

[66] S. Huang, H. Fu, Z. Yuan, A. Vaivads, Y. V. Khotyaintsev, A. Retino, M. Zhou, D. B. Graham, K. Fujimoto, F. Sahraoui, et al., Journal of Geophysical Research: 
Space Physics 121, 6639 (2016).

[67] C. Bertucci, N. Achilleos, C. Mazelle, G. B. Hospodarsky, M. Thomsen, M. K. Dougherty, and W. Kurth, J. Geophys. Res. 112, 219 (2007).

[68] N. Andrés, D. O. Gómez, C. Bertucci, C. Mazelle, and M. K. Dougherty, Planetary and Space Science 79-80, 64 (2013)

[69] N. Andrés, K. Meziane, C. Mazelle, C. Bertucci, and D. O. Gómez, Journal of Geophysical Research: Space Physics 120, 4181 (2015), 2014JA020783.

[70] J. Cho and A. Lazarian, Phys. Rev. Lett. 88, 245001 (2002).

[71] Y. Yang, W. H. Matthaeus, Y. Shi, M. Wan, and S. Chen, Physics of Fluids 29, 035105 (2017).

[72] F. Saharoui, S. Galtier, and G. Belmont, Journal of Plasma Physics 73, 723-730 (2007).

[73] C. W. Smith, B. J. Vasquez, and K. Hamilton, J. Geophys. Res. 111, A09111 (2006).

[74] S. Oughton, W. H. Matthaeus, M. Wan, and T. Parashar, J. Geophys. Res. 121, 5041 (2016).

[75] E. A. Kuznetsov, Journal of Experimental and Theoretical Physics 93, 1052 (2001).

[76] B. D. G. Chandran, Phys. Rev. Lett. 101, 235004 (2008).

[77] B. D. G. Chandran, Phys. Rev. Lett. 95, 265004 (2005).

[78] P. Clark di Leoni, P. J. Cobelli, and P. D. Mininni, The European Physical Journal E 38, 136 (2015a).

[79] R. Fitzpatrick, Plasma Physics: An Introduction (CRC Press, 2014).

[80] N. A. Krall and A. W. Trivelpiece, in Principle of Plasma Physics , 89 (1973).

[81] D. O. Gómez, P. D. Mininni, and P. Dmitruk, Phys. Scripta T116 123 (2005).

[82] P. D. Mininni, D. Rosenberg, R. Reddy, and A. Pouquet, Parallel Computing 37, 16 (2011).
[83] S. Ghosh, M. Hossain, and W. H. Matthaeus, Computer Phys. Commun. 74, 18 (1993).

[84] P. Dmitruk, W. H. Matthaeus, and S. Oughton, Phys. Plasmas 12, 112304 (2005).

[85] F. Sahraoui, G. Belmont, and L. Rezeau, Physics of Plasmas 10, 1325 (2003).

[86] P. Clark di Leoni, P. J. Cobelli, P. D. Mininni, P. Dmitruk, and W. M. Matthaeus, Phys. Fluids 26, 035106 (2014).

[87] P. Clark di Leoni and P. D. Mininni, Phys. Rev. E 91, 033015 (2015c).

[88] P. Clark di Leoni, P. D. Mininni, and M. E. Brachet, Phys. Rev. A 92, 063632 (2015b).

[89] R. Lugones, P. Dmitruk, P. D. Mininni, M. Wan, and W. H. Matthaeus, submitted.

[90] F. Sahraoui, M. L. Goldstein, G. Belmont, P. Canu, and L. Rezeau, Phys. Rev. Lett. 105, 131101 (2010).

[91] W. H. Matthaeus and M. L. Goldstein, Phys. Rev. Lett. 57, 495 (1986).

[92] M. Velli, R. Grappin, and A. Mangeney, Physical Review Letters 63, 1807 (1989).

[93] W. Matthaeus, B. Breech, P. Dmitruk, A. Bemporad, G. Poletto, M. Velli, and M. Romoli, The Astrophysical Journal Letters 657, L121 (2007).

[94] P. Dmitruk, P. D. Mininni, A. Pouquet, S. Servidio, and W. H. Matthaeus, Phys. Rev. E 83, 066318 (2011).

[95] P. Dmitruk, P. D. Mininni, A. Pouquet, S. Servidio, and W. H. Matthaeus, Phys. Rev. E 90, 043010 (2014).

[96] D. D. Joseph, Proceedings of the National Academy of Sciences 103, 14272 (2006).

[97] P. L. Sulem, T. Passot, D. Laveder, and D. Borgogno, The Astrophysical Journal 818, 66 (2016). 